\title{
Some Integral Inequalities of Hermite-Hadamard Type for $\varepsilon$-convex Functions
}

\author{
Jun Zhang' ${ }^{1}$ Zhi-Li Pei ${ }^{1}$, Feng $\mathbf{Q i}^{2,3, *}$ \\ ${ }^{1}$ College of Computer Science and Technology, Inner Mongolia University for Nationalities, Tongliao City 028043, \\ Inner Mongolia Autonomous Region, China \\ ${ }^{2}$ School of Mathematics and Informatics, Henan Polytechnic University, Jiaozuo City, Henan Province, 454010, China, \\ ${ }^{3}$ Department of Mathematics, School of Science, Tianjin Polytechnic University, Tianjin City, 300387, China \\ *Corresponding author: qifeng618@gmail.com
}

Received January 07, 2017; Revised May 04, 2017; Accepted May 19, 2017

\begin{abstract}
In the paper, the authors establish a new integral identity. By this integral identity and Hölder's inequality, the authors obtain some new inequalities of the Hermite-Hadamard type for $\varepsilon$-convex functions.
\end{abstract}

Keywords: Hermite-Hadamard type type inequality, Integral identity, ع-convex function

Cite This Article: Jun Zhang, Zhi-Li Pei, and Feng Qi, "Some Integral Inequalities of Hermite-Hadamard Type for $\varepsilon$-convex Functions.” Turkish Journal of Analysis and Number Theory, vol. 5, no. 3 (2017): 117-120. doi: 10.12691/tjant-5-3-5.

\section{Introduction}

We first list some definitions concerning various convex functions.

Definition 1.1. A function $f: I \subseteq R=(-\infty, \infty) \rightarrow R$ is said to be convex if

$$
f(\lambda x+(1-\lambda) y) \leq \lambda f(x)+(1-\lambda) f(y)
$$

holds for all $x, y \in I$ and $\lambda \in[0,1]$.

In [1] the concept of $\varepsilon$-convex functions was introduced as follows.

Definition 1.2. [1] A function $f: I \subseteq R=(-\infty, \infty) \rightarrow R$ and $\varepsilon \geq 0$, if

$$
f(\lambda x+(1-\lambda) y) \leq \lambda f(x)+(1-\lambda) f(y)+\varepsilon,
$$

is valid for all $x, y \in I$ and $\lambda \in[0,1]$, then we say that $f(x)$ is a $\varepsilon$-convex function on $I$.

The following inequalities of Hermite-Hadamard type were established for some of the above convex functions.

Theorem 1.1. [2] Let $f: I^{\circ} \subseteq R \rightarrow R$ be differentiable on $I^{\circ}, a, b \in I^{\circ}$ with $a<b$.

(i) If $\left|f^{\prime}\right|$ is convex function on $[a, b]$, then

$$
\begin{aligned}
& \left|\frac{f(a)+f(b)}{2}-\frac{1}{b-a} \int_{a}^{b} f(x) d x\right| \\
& \leq \frac{(b-a)\left(\left|f^{\prime}(a)\right|+\left|f^{\prime}(b)\right|\right)}{8} .
\end{aligned}
$$

(ii) If $\left|f^{\prime}\right|^{p /(p-1)}$ is convex function on $[a, b], p>1$, then

$$
\begin{aligned}
& \left|\frac{f(a)+f(b)}{2}-\frac{1}{b-a} \int_{a}^{b} f(x) d x\right| \\
& \leq \frac{b-a}{2(p+1)^{1 / p}}\left(\frac{\left|f^{\prime}(a)\right|^{p /(p-1)}+\left|f^{\prime}(b)\right|^{p /(p-1)}}{2}\right)^{(p-1) / p} .
\end{aligned}
$$

Theorem 1.2. [3] Let $f: I^{\circ} \subseteq R \rightarrow R$ be differentiable on $I^{\circ}, a, b \in I^{\circ}$ with $a<b$ and $q \geq 1$.

(i) If $|f|^{q}$ is convex function on $[a, b]$, then

$$
\begin{aligned}
& \left|f\left(\frac{a+b}{2}\right)-\frac{1}{b-a} \int_{a}^{b} f(x) d x\right| \\
& \leq \frac{b-a}{4}\left[\frac{\left|f^{\prime}(a)\right|^{q}+\left|f^{\prime}(b)\right|^{q}}{2}\right]^{1 / q} .
\end{aligned}
$$

(ii) If $|f|^{q}$ is convex function on $[a, b], p>1$, then

$$
\left|f\left(\frac{a+b}{2}\right)-\frac{1}{b-a} \int_{a}^{b} f(x) d x\right| \leq \frac{b-a}{4}\left|f^{\prime}\left(\frac{a+b}{2}\right)\right|,(1.6
$$

and

$$
\begin{aligned}
& \left|\frac{f(a)+f(b)}{2}-\frac{1}{b-a} \int_{a}^{b} f(x) d x\right| \\
& \leq \frac{b-a}{4}\left|f^{\prime}\left(\frac{a+b}{2}\right)\right| .
\end{aligned}
$$

In this paper, we establish a new integral identity. By this identity and Hölder's inequality, some new HermiteHadamard type for the product of $\varepsilon$-convex function and discussed, some results are obtained. 


\section{Some Lemmas}

Lemma 2.1 Let $f: I \subseteq R \rightarrow R$ be differentiable on $I^{\circ}$ and where $a, b \in I$ with $a<b, n \in N_{+}$. If $f^{\prime} \in L_{1}([a, b])$, then the following identity holds:

$$
\begin{aligned}
& \frac{1}{n+1}\left[f(a)+\sum_{k=2}^{n} f\left(a+\frac{(k-1)(b-a)}{n}\right)+f(b)\right] \\
& -\frac{1}{b-a} \int_{a}^{b} f(x) \mathrm{d} x \\
& =\frac{b-a}{n^{2}} \sum_{k=1}^{n} \int_{0}^{1}\left[\begin{array}{l}
\left(t-\frac{n-(k-1)}{n+1}\right) \\
\left.f^{\prime}\left(\begin{array}{l}
(1-t)\left(a+\frac{(k-1)(b-a)}{n}\right) \\
+t\left(a+\frac{k(b-a)}{n}\right)
\end{array}\right)\right] \mathrm{d} t .
\end{array}\right.
\end{aligned}
$$

Proof.

$$
\begin{gathered}
\left.\frac{b-a}{n^{2}} \sum_{k=1}^{n} \int_{0}^{1}\left[\begin{array}{l}
\left(t-\frac{n-(k-1)}{n+1}\right) \\
f^{\prime}\left(\begin{array}{l}
(1-t)\left(a+\frac{(k-1)(b-a)}{n}\right) \\
+t\left(a+\frac{k(b-a)}{n}\right)
\end{array}\right)
\end{array}\right)\right] \mathrm{d} t \\
=\frac{1}{n} \sum_{k=1}^{n}\left[\frac{n-(k-1)}{n+1} f\left(a+\frac{(k-1)(b-a)}{n}\right)\right. \\
+\frac{k}{n+1} f\left(a+\frac{k(b-a)}{n}\right) \\
-\int_{0}^{1} f\left(\begin{array}{l}
\left.(1-t)\left(a+\frac{(k-1)(b-a)}{n}\right)\right) \\
+t\left(a+\frac{k(b-a)}{n}\right)
\end{array}\right) .
\end{gathered}
$$

Since

$$
\begin{aligned}
& \frac{1}{n} \sum_{k=1}^{n}\left[\begin{array}{l}
\frac{n-(k-1)}{n+1} f\left(a+\frac{(k-1)(b-a)}{n}\right) \\
+\frac{k}{n+1} f\left(a+\frac{k(b-a)}{n}\right)
\end{array}\right] \\
& =\frac{1}{n}\left[\frac{n}{n+1} f(a)+\frac{1}{n+1} f\left(a+\frac{b-a}{n}\right)\right. \\
& +\frac{n-1}{n+1} f\left(a+\frac{b-a}{n}\right)+\frac{2}{n+1} f\left(a+\frac{2(b-a)}{n}\right) \\
& +\frac{n-2}{n+1} f\left(a+\frac{2(b-a)}{n}\right)+\frac{3}{n+1} f\left(a+\frac{3(b-a)}{n}\right) \\
& +\frac{n-3}{n+1} f\left(a+\frac{3(b-a)}{n}\right)+\frac{4}{n+1} f\left(a+\frac{4(b-a)}{n}\right) \\
& +\frac{2}{n+1} f\left(a+\frac{(n-2)(b-a)}{n}\right)+\frac{n-1}{n+1} f\left(a+\frac{(n-1)(b-a)}{n}\right)
\end{aligned}
$$

$$
\begin{aligned}
& \left.+\frac{1}{n+1} f\left(a+\frac{(n-1)(b-a)}{n}\right)+\frac{n}{n+1} f(b)\right] \\
= & \frac{1}{n+1}\left[f(a)+\sum_{k=2}^{n} f\left(a+\frac{(k-1)(b-a)}{n}\right)+f(b)\right] .
\end{aligned}
$$

Changing variables with

$$
x=(1-t)\left(a+\frac{(k-1)(b-a)}{n}\right)+t\left(a+\frac{k(b-a)}{n}\right)
$$

for $0 \leq t \leq 1$, we get

$$
\begin{aligned}
& \frac{1}{n} \sum_{k=1}^{n} \int_{0}^{1} f\left(\begin{array}{c}
(1-t)\left(a+\frac{(k-1)(b-a)}{n}\right) \\
+t\left(a+\frac{k(b-a)}{n}\right)
\end{array}\right) \mathrm{d} t \\
= & \frac{1}{n} \sum_{k=1}^{n} \frac{n}{b-a} \int_{a+\frac{(k-1)(b-a)}{n}}^{a+\frac{k(b-a)}{n}} f(x) \mathrm{d} x \\
= & \frac{1}{b-a} \int_{a}^{b} f(x) \mathrm{d} x .
\end{aligned}
$$

Put the equalities (2.3) to (2.4) into the equality (2.2), the inequality (2.1) is thus proved. This completes of the proof.

By taking $n=1$ in Lemma 2.1, we have the following identities.

Lemma 2.2 [2] Let $f: I \subseteq R \rightarrow R$ be differentiable on $I^{\circ}$ and where $a, b \in I$ with $a<b$, if $f^{\prime} \in L_{1}([a, b])$, then

$$
\begin{aligned}
& \frac{1}{b-a} \int_{a}^{b} f(x) \mathrm{d} x-\frac{f(a)+f(b)}{2} \\
& =\frac{b-a}{2} \int_{0}^{1}(1-2 t) f^{\prime}(t a+(1-t) b) \mathrm{d} t .
\end{aligned}
$$

\section{Some Integral Inequalities of Hermite-Hadamard Type}

Now we are in a position to establish some new integral inequalities of Hermite-Hadamard type involving the $\varepsilon$ convex functions.

Theorem 3.1 Let $f:[a, b] \subseteq R \rightarrow R$ be differentiable function on $[a, b]$ and $\varepsilon \geq 0, n \in N_{+}, q \geq 1$. If $f^{\prime} \in L_{1}([a, b])$, then

$$
\begin{aligned}
& \left|\begin{array}{l}
\frac{1}{n+1}\left[f(a)+\sum_{k=2}^{n} f\left(a+\frac{(k-1)(b-a)}{n}\right)+f(b)\right] \\
-\frac{1}{b-a} \int_{a}^{b} f(x) \mathrm{d} x
\end{array}\right| \\
& \leq \frac{(b-a)(2 n+1)}{6 n(n+1)}\left[\frac{\left|f^{\prime}(a)\right|^{q}+\left|f^{\prime}(b)\right|^{q}}{2}+\varepsilon\right]^{1 / q} \text {. }
\end{aligned}
$$

Proof. Since $\left|f^{\prime}\right|^{q}$ is $\varepsilon$-convex function on $[a, b]$, using the Lemma 2.1 and by the Hölder's inequality, we have 


$$
\begin{aligned}
& \left|\begin{array}{l}
\frac{1}{n+1}\left[f(a)+\sum_{k=2}^{n} f\left(a+\frac{(k-1)(b-a)}{n}\right)+f(b)\right] \\
-\frac{1}{b-a} \int_{a}^{b} f(x) \mathrm{d} x
\end{array}\right| \\
& \leq \frac{b-a}{n^{2}} \sum_{k=1}^{n} \int_{0}^{1}\left|t-\frac{n-k+1}{n+1}\right| \\
& \left.\times \mid \begin{array}{l}
f^{\prime}(1-t)\left(a+\frac{(k-1)(b-a)}{n}\right) \\
+t\left(a+\frac{k(b-a)}{n}\right)
\end{array}\right) \mid \mathrm{d} t \\
& \leq \frac{b-a}{n^{2}}\left(\sum_{k=1}^{n} \int_{0}^{1}\left|t-\frac{n-k+1}{n+1}\right| \mathrm{d} t\right)^{1-1 / q} \\
& \left(\sum_{k=1}^{n} \int_{0}^{1}\left|t-\frac{n-k+1}{n+1}\right|\right. \\
& \times\left.\left|f^{\prime}\left(\begin{array}{l}
(1-t)\left(a+\frac{(k-1)(b-a)}{n}\right) \\
+t\left(a+\frac{k(b-a)}{n}\right)
\end{array}\right)\right|^{q} \mathrm{~d} t\right|^{1 / q} \\
& \leq \frac{b-a}{n^{2}}\left(\sum_{k=1}^{n} \int_{0}^{1}\left|t-\frac{n-k+1}{n+1}\right| \mathrm{d} t\right)^{1-1 / q} \\
& {\left[\sum_{k=1}^{n} \int_{0}^{1}\left|t-\frac{n-k+1}{n+1}\right|\right.} \\
& \left.\times\left(\begin{array}{c}
\frac{n-k+1-t}{n}\left|f^{\prime}(a)\right|^{q} \\
+\frac{k-1+t}{n}\left|f^{\prime}(b)\right|^{q}+\varepsilon
\end{array}\right) \mathrm{d} t\right]^{1 / q} \\
& =\frac{b-a}{n^{2}}\left(\frac{n(2 n+1)}{6(n+1)}\right)^{1-1 / q} \\
& \times\left[\begin{array}{l}
\frac{n(2 n+1)\left[\left|f^{\prime}(a)\right|^{q}+\left|f^{\prime}(b)\right|^{q}\right]}{12(n+1)} \\
+\varepsilon \frac{n(2 n+1)}{6(n+1)}
\end{array}\right]^{1 / q} \\
& =\frac{(b-a)(2 n+1)}{6 n(n+1)}\left[\frac{\left|f^{\prime}(a)\right|^{q}+\left|f^{\prime}(b)\right|^{q}}{2}+\varepsilon\right]^{1 / q} .
\end{aligned}
$$

Theorem 3.1 is proved.

Crollary3.1. Under the conditions of Theorem 3.1, then

(1) if $n=1$, we have

$$
\begin{aligned}
& \left|\frac{f(a)+f(b)}{2}-\frac{1}{b-a} \int_{a}^{b} f(x) \mathrm{d} x\right| \\
& \leq \frac{b-a}{4}\left[\frac{\left|f^{\prime}(a)\right|^{q}+\left|f^{\prime}(b)\right|^{q}}{2}+\varepsilon\right]^{1 / q} .
\end{aligned}
$$

(2) if $q=1$, we have

$$
\begin{aligned}
& \mid \begin{array}{l}
\frac{1}{n+1}\left[f(a)+\sum_{k=2}^{n} f\left(a+\frac{(k-1)(b-a)}{n}\right)+f(b)\right] \\
-\frac{1}{b-a} \int_{a}^{b} f(x) \mathrm{d} x
\end{array} \\
& \leq \frac{(b-a)(2 n+1)}{6 n(n+1)}\left[\frac{\left|f^{\prime}(a)\right|+\left|f^{\prime}(b)\right|}{2}+\varepsilon\right] .
\end{aligned}
$$

Theorem 3.2 Let $f:[a, b] \subseteq R \rightarrow R$ be differentiable function on $[a, b]$ and $\varepsilon \geq 0, n \in N_{+}, q>1$ and $0 \leq r \leq q$. If $f^{\prime} \in L_{1}([a, b])$, then

$$
\begin{aligned}
& \left|\begin{array}{l}
\frac{1}{n+1}\left[f(a)+\sum_{k=2}^{n} f\left(a+\frac{(k-1)(b-a)}{n}\right)+f(b)\right. \\
-\frac{1}{b-a} \int_{a}^{b} f(x) \mathrm{d} x
\end{array}\right| \\
& \leq \frac{b-a}{n^{2}}\left[\frac{q-1}{2 q-r-1} \sum_{k=1}^{n}\left(\begin{array}{l}
\left(\frac{k}{n+1}\right)^{\frac{2 q-r-1}{q-1}} \\
+\left(1-\frac{k}{n+1}\right)^{\frac{2 q-r-1}{q-1}}
\end{array}\right)\right]^{1-1 / q} \\
& \times\left\{\sum _ { k = 1 } ^ { n } \left[\left(\frac{k}{n+1}\right)^{r} n[n(r+2)+r+1] k^{2}\left|f^{\prime}(a)\right|^{q}\right.\right. \\
& +\left(1-\frac{k}{n+1}\right)^{r}[(n(r+2)+r+1) k \\
& \left.\left.-(n+1)(r+1)]\left|f^{\prime}(b)\right|^{q}\right]\right\}^{1 / q} .
\end{aligned}
$$

Proof. Since $\left|f^{\prime}\right|^{q}$ is $\varepsilon$-convex function on $[a, b]$, using the Lemma 2.1 and by the Hölder's inequality, we have

$$
\begin{aligned}
& \left|\begin{array}{l}
\frac{1}{n+1}\left[f(a)+\sum_{k=2}^{n} f\left(a+\frac{(k-1)(b-a)}{n}\right)+f(b)\right] \\
-\frac{1}{b-a} \int_{a}^{b} f(x) \mathrm{d} x
\end{array}\right| \\
& \leq \frac{b-a}{n^{2}} \sum_{k=1}^{n} \int_{0}^{1}\left|t-\frac{n-k+1}{n+1}\right| \\
& \times\left|f^{\prime}\left((1-t)\left(a+\frac{(k-1)(b-a)}{n}\right)+t\left(a+\frac{k(b-a)}{n}\right)\right)\right| \mathrm{d} t \\
& \leq \frac{b-a}{n^{2}}\left(\sum_{k=1}^{n} \int_{0}^{1}\left|t-\frac{n-k+1}{n+1}\right|^{(q-r) /(q-1)} \mathrm{d} t\right)^{1-1 / q} \\
& \times\left(\sum_{k=1}^{n} \int_{0}^{1}\left|t-\frac{n-k+1}{n+1}\right|^{r}\right. \\
& \times\left.\left|f^{\prime}\left(\begin{array}{l}
(1-t)\left(a+\frac{(k-1)(b-a)}{n}\right) \\
+t\left(a+\frac{k(b-a)}{n}\right)
\end{array}\right)\right| d t\right|^{q}
\end{aligned}
$$




$$
\begin{aligned}
\leq & \left.\frac{b-a}{n^{2}}\left|\sum_{k=1}^{n} \int_{0}^{1}\right| t-\left.\frac{n-k+1}{n+1}\right|^{(q-r) /(q-1)} \mathrm{d} t\right)^{1-1 / q} \\
& \times\left[\sum_{k=1}^{n} \int_{0}^{1}\left|t-\frac{n-k+1}{n+1}\right|^{r}\right. \\
& \left.\times\left(\frac{n-k+1-t}{n}\left|f^{\prime}(a)\right|^{q}+\frac{k-1+t}{n}\left|f^{\prime}(b)\right|^{q}+\varepsilon\right) \mathrm{d} t\right]^{1 / q} \\
\leq & \left.\frac{b-a}{n^{2}}\left[\frac{q-1}{2 q-r-1} \sum_{k=1}^{n} \mid\left(\frac{k}{n+1}\right)^{\frac{2 q-r-1}{q-1}}\right)\right|^{1-1 / q} \\
& \times\left\{\sum _ { k = 1 } ^ { n } \left[\left(\frac{k}{n+1}\right)^{r} n[n(r+2)+r+1] k^{2}\left|f^{\prime}(a)\right|^{q}\right.\right. \\
& +\left(1-\frac{k}{n+1}\right)^{\frac{2 q-r-1}{q-1}}[(n(r+2)+r+1) k \\
& \left.\left.-(n+1)(r+1)]\left|f^{\prime}(b)\right|^{q}\right]\right\}^{1 / q} \cdot \\
& (1-1 \\
& (n+3)
\end{aligned}
$$

Theorem 3.2 is proved.

\section{Acknowledgements}

This work was supported by the National Natural Science Foundation under Grant No. 61373067 and No.
61672301 of China and by the Inner Mongolia Autonomous Region Natural Science Foundation Project under Grant No. 2015MS0123, China.

\section{References}

[1] D. H. Hyers, S. M. Ulam, Approximately convex functions, Proc Amer. Math. Soc., 3 (1952), 821-828.

[2] S. S. Dragomir, and R. P. Agarwal, Two inequalities for differentiable mappings and applications to special means of real numbers and to trapezoidal formula, Appl. Math. Lett., 11(1998), 91-95.

[3] C. E. M. Pearce and J. Pečarić, Inequalities for differentiable mappings with application to special means and quadrature formula. Appl. Math. Lett., 13(2000), 51-55.

[4] F. Qi and B.-Y. Xi, Some Hermite-Hadamard type inequalities for geometrically quasi-convex functions, Proceedings of the Indian Academy of Sciences (Mathematical Sciences), 124:3(2014), 333-342.

[5] F. Qi, T.-Y. Zhang, and B.-Y. Xi, Integral inequalities of HermiteHadamard type for functions whose first derivatives are of convexity, Ukrainian Mathematical Journal, 66:5(2015), 625-640.

[6] B.-Y. Xi, R.-F. Bai, and F. Qi, Hermite-Hadamard type inequalities for the $m$ - and $(\alpha, m)$-geometrically convex functions, Aequationes Math., 184:3(2012), 261-269.

[7] B.-Y. Xi and F. Qi, Hermite-Hadamard type inequalities for geometrically $r$-convex functions, Studia Scientiarum Mathematicarum Hungarica, 51:4(2014), 530-546;

[8] B.-Y. Xi and F. Qi, Inequalities of Hermite-Hadamard type for extended s-convex functions and applications to means, J. Nonlinear Convex Anal,. 16(2015), 873-890.

[9] B.-Y. Xi, S.-H. Wang, and F. Qi, Some inequalities for $(h, m)$-convex functions, Journal of Inequalities and Applications, 2014, 100, 12 pages.

[10] B.-Y. Xi, T.-Y. Zhang, and F. Qi, Some inequalities of Hermite-Hadamard type for $m$-harmonic-arithmetically convex functions, ScienceAsia, 41: 5(2015), 357-361. 